\title{
THE IRREGULARITY OF AN ALGEBRAIC SURFACE AND A THEOREM ON REGULAR SURFACES
}

\author{
H. T. MUHLY 1
}

1. Introduction. In a recent paper $[3]^{2} \mathrm{O}$. Zariski and the writer have shown that the arithmetic genus of a field $\Sigma$ of algebraic functions of two variables can be invariantly defined with the aid of the Hilbert characteristic function associated with a pair of models of $\Sigma$. The first objective of this note is to show that the irregularity of $\Sigma$ can be defined in a similar manner. The second objective is to obtain more general results on the existence of integral bases for regular surfaces than were obtained in [2].

2. The irregularity. We consider a field $\Sigma$ of algebraic functions of two independent variables over a ground field $k$. The field $k$ is assumed to be of characteristic zero and to be maximally algebraic in $\Sigma$. We use the notations and definitions of [3]. In particular, if $U$ and $V$ are normal models of $\Sigma$ we let $\left\{A_{m}\right\}\left(\left\{B_{n}\right\}\right)$ denote the system of curves cut out on $U(V)$ by the hypersurfaces of order $m(n)$ of its ambient space. The dimension increased by one of the complete system $\left|A_{m}+B_{n}\right|$ (which system is regarded as lying on the join $W$ of $U$ and $V)$ is denoted by $r(m, n)$. The transformation $T: U \rightarrow V$ is said to be proper [3, definition 2] if $T(A)$ is normal for a generic $A \in\left\{A_{1}\right\}$.

Lemma 1. If the transformation $T: U \rightarrow V$ is proper then there exists an integer $n_{0}$ such that for $n \geqq n_{0}$ and $m \geqq i$ the complete system $\left|A_{m}+B_{n}\right|$ cuts a complete series on the generic curve $A_{i}$ of the system $\left|A_{i}\right|$, where $i$ is an arbitrary positive integer.

Proof. Let $A$ be a nonsingular irreducible hyperplane section of $U$ such that $T(A)$ is normal. (Such hyperplane sections exist in view of the Bertini-Zariski theorems [7] and [9] and the fact that $T$ is proper.) If $\bar{r}(m, n)$ is the $r$-function associated with the pair $(A, T(A))$ in the sense of [3] (article 2), then by formula 4.1 of [3], there exists an integer $n_{0}$ such that when $n \geqq n_{0}$ the function $r(m, n)$ satisfies the addition formula

$$
r(m, n)=r(m-1, n)+\bar{r}(m, n) .
$$

Presented to the Society, September 10, 1948; received by the editors July 20,1948.

1 Research paper done under Office of Naval Research contract N6ori-71, Task Order XVIII (NR-043 054) at the University of Illinois and Harvard University. The ideas expressed in this paper represent the personal views of the author and are not necessarily those of the Office of Naval Research.

${ }^{2}$ Numbers in brackets refer to the bibliography. 
If $\pi$ is the genus of $A$, if $\mu$ and $\nu$ are respectively the orders of $A$ and $T(A)$, and if $n \nu>2 \pi-2$, then the function $\bar{r}(m, n)$ is given by the formula $\bar{r}(m, n)=m \mu+n \nu-\pi+1$, so that by repeated application of (2.1),

$$
r(m, n)=r(m-i, n)+i(m \mu+n \nu-\pi+1)-2^{-1} i(i-1) \mu .
$$

If $\mu_{i}$ and $\pi_{i}$ are the degree and genus of the system $\left|A_{i}\right|$ the well known formulas of Noether yield

$$
\begin{aligned}
& \mu_{i}=i^{2} \mu, \\
& \pi_{i}=i \pi+2^{-1} i(i-1) \mu-i+1,
\end{aligned}
$$

so that (2.2) becomes

$$
r(m, n)=r(m-i, n)+i(m \mu+n \nu)-\pi_{i}+1 .
$$

Let $A_{i}$ be a generic ${ }^{3}$ member of $\left|A_{i}\right|$ and observe that $\left|A_{m}+B_{n}\right|$ cuts a series of order $(m \mu+n \nu) i$ on $A_{i}$. If necessary we increase $n_{0}$ so that $n_{0} \nu>2 \pi-2$. It then follows from the second of formulas (2.3) that if $m \geqq i$ and $n \geqq n_{0}$ the inequality, $(m \mu+n v) i>2 \pi_{i}-2$, holds so that the series cut on $A_{i}$ by $\left|A_{m}+B_{n}\right|$ is non-special. If $d$ is its deficiency, then the dimension of this series is $(m \mu+n \nu) i-\pi_{i}-d$, and since the residual system of $\left|A_{m}+B_{n}\right|$ with respect to $A_{i}$ is the complete system $\left|A_{m-i}+B_{n}\right|$,

$$
r(m, n)=r(m-i, n)+(m \mu+n v) i-\pi_{i}-d+1 .
$$

Equations (2.4) and (2.5) together imply that $d=0$, q.e.d.

It is well known that if $C_{1}$ and $C_{2}$ are generic members of a linear system $|C|$ of curves on an algebraic surface then the characteristic series cut by $|C|$ on $C_{i}(i=1,2)$ will have the same deficiency. This deficiency is therefore a character of the system $|C|$. We quote the following lemma proved in [4] (page 70).

LEMMA 2. If the system $|C+D|$ cuts out a complete series on a generic curve $C \in|C|$, then the deficiency of the characteristic series of $|C|$ is not greater than that of $|D|$.

If we let $\alpha(m)$ and $\beta(n)$ denote the deficiencies of the characteristic series of the complete systems $\left|A_{m}\right|$ and $\left|B_{n}\right|$ respectively then Lemmas 1 and 2 together yield the following theorem.

Theorem 1. If the transformation $T: U \rightarrow V$ is proper then there exists

${ }^{3}$ The term "generic" is here used only to signify that $A_{i}$ is irreducible and has genus $\pi_{i}$, where $\pi_{i}$ is the genus of $\left|A_{i}\right|$. Unless otherwise specified the term will be used in this sense throughout the text. 
an integer $n_{0}$ such that $\alpha(m) \leqq \beta(n)$ when $n \geqq n_{0}$ and $m$ is arbitrary.

Proof. By Lemma 1 there is an integer $n_{0}$ such that $\left|A_{m}+B_{n}\right|$ cuts a complete series on the generic curve of $\left|A_{m}\right|$ when $n \geqq n_{0}$ and $m$ is arbitrary. By Lemma 2 it follows that $\alpha(m) \leqq \beta(n)$, q.e.d.

CoRollary 1. If both $T: U \rightarrow V$ and $T^{-1}: V \rightarrow U$ are proper then there exist integers $m_{0}$ and $n_{0}$ such that $\alpha(m)=\beta(n)$ if $m \geqq m_{0}$ and $n \geqq n_{0}$.

It follows that the deficiency $\alpha(m)$ of the characteristic series of the complete system determined by the $m$-fold of the system of hyperplane sections of any normal model $U$ of $\Sigma$ is independent of $m$ if $m$ is sufficiently large. Indeed, if $V$ is any model of $\Sigma$ in regular correspondence with $U$ (so that both $T: U \rightarrow V$ and $T^{-1}: V \rightarrow U$ are proper [3]) and if $m_{0}$ and $n_{0}$ are the integers determined by the pair $(U, V)$ in the sense of Corollary 1 , then $\alpha(m)=\beta\left(n_{0}\right)$ for all $m \geqq m_{0}$. Moreover, $\alpha\left(m_{0}\right)$ is the maximum value of $\alpha(m)$ since $\alpha(m) \leqq \beta\left(n_{0}\right)=\alpha\left(m_{0}\right)$ for all $m$. This maximum value assumed by the function $\alpha(m)$ is therefore a character of the model $U$. We denote it by $\delta(U)$. The non-negative numerical character $\delta(U)$ is a relative invariant of $U$, for if $U$ and $V$ are in regular correspondence then $\delta(U)=\alpha\left(m_{0}\right)=\beta\left(n_{0}\right)$ $=\delta(V)$.

COROLlARy 2. If $U$ and $V$ are normal models of $\Sigma$ such that $U<V$ then $\delta(U) \leqq \delta(V)$.

Proof. If $U$ and $V$ are normal and if $U<V$ then $T: U \rightarrow V$ is proper. Hence by Theorem 1 there exists an integer $n_{0}$ such that $\delta(U) \leqq \beta\left(n_{0}\right) \leqq \delta(V)$, q.e.d.

COROLlaRY 3. If $U$ and $V$ are nonsingular models of $\Sigma$ then $\delta(U)$ $=\delta(V)$.

Proof. It is shown in [8] that there exist models $U_{1}$ and $V_{1}$ such that the correspondence $T_{1}: U_{1} \rightarrow V_{1}$ is regular and such that $U_{1}\left(V_{1}\right)$ is obtained from $U(V)$ by a sequence of quadratic transformations with simple centers. Since such quadratic transformations and their inverses are proper [3, Lemma 3] and since $T_{1}$ and $T_{1}^{-1}$ are proper Corollary 1 implies that $\delta(U)=\delta\left(U_{1}\right)=\delta\left(V_{1}\right)=\delta(V)$, q.e.d.

Since the character $\delta(U)$ has the same value for all nonsingular models of $\Sigma$ we can regard it as a character of $\Sigma$. It is this character

4 The notation $U<V$ is used to indicate that the birational transformation $T^{-\mathbf{1}}$ : $V \rightarrow U$ has no fundamental points on $V$; or equivalently, the local ring $Q\left(P^{\prime}\right)$ contains the local ring $Q(P)$, when $P(\subset U)$ and $P^{\prime}(C V)$ are a pair of corresponding points in the birational correspondence $T$. 
which we define to be the irregularity $q$ of the field $\Sigma$, and $\Sigma$ is said to be regular or irregular according as $q=0$, or $q>0$. The use of the term "irregularity" for this character of $\Sigma$ is justified by the well known work of Castelnuovo (see [4, chap. IV]) who has obtained the above results by different methods.

Corollary 4. If $U$ is any normal model of $\Sigma$ then $\delta(U) \leqq q$.

Proof. There exists a nonsingular model $V$ such that $U<V$ (see [6]). Hence $\delta(U) \leqq \delta(V)=q$, q.e.d.

3. Regular models. Let $U$ be a normal model of $\Sigma$, and let $\left\{A_{m}\right\}$ be the system cut out on $U$ by the hypersurfaces of order $m$ of its ambient space.

Lemma 3. There exists an integer $n_{0}$ such that if $n \geqq n_{0}$ the complete system $\left|r A_{n}\right|$ cuts out a complete series on the generic curve of $\left|A_{n}\right|$ for any integer $r \geqq 2$.

Proof. We regard $U$ as being in regular birational correspondence with itself under the identity correspondence and we identify the systems $\left\{A_{m}\right\}$ and $\left\{B_{m}\right\}$ of the preceding article. By Lemma 1 there exists an integer $n_{0}$ such that $\left|A_{m+n}\right|$ cuts a complete series on a generic $A_{m} \in\left|A_{m}\right|$ when $n \geqq n_{0}$. It follows that $\left|A_{n+n s}\right|$ cuts a complete series on a generic $A_{n}$ if $n \geqq n_{0}$ and $s$ is an arbitrary positive integer. Hence $\left|A_{r n}\right|\left(=\left|r A_{n}\right|\right)$ cuts a complete series on $A_{n}$ if $n \geqq n_{0}$ and $r \geqq 2$, q.e.d.

THEOREM 2. If $U$ is a normal model of $\Sigma$ such that the relative invariant $\delta(U)$ is zero, then there exists an integer $h_{0}$ such that if $U_{h}$ is any derived arithmetically normal model of $U$ belonging to a character of homogeneity $h \geqq h_{0}$, then the generic hyperplane section of $U_{h}$ is arithmetically normal. In fact, any irreducible nonsingular hyperplane section of $U_{h}$ is an arithmetically normal curve.

Proof. Let $h_{0}$ be the integer $n_{0}$ determined in Lemma 3 and let $h$ be a character of homogeneity ${ }^{5}$ of $U$ such that $h \geqq h_{0}$. If $\left\{A_{n}\right\}$ denotes as usual the system cut out on $U$ by the hypersurfaces of order $n$ of its ambient space, and if $U_{h}$ is the derived arithmetically normal model of $U$ belonging to the character $h$, then the hypersurfaces of order $r$ in the ambient space of $U_{h}$ will cut out the complete system $\left|A_{h r}\right|$ on $U_{h}, r=1,2, \cdots[1]$. Since $h \geqq h_{0}$, it follows that $\left|A_{h r}\right|$ will cut out a complete series on the generic curve of $\left|A_{h}\right|$ if $r \geqq 2$. If necessary,

${ }^{6}$ For the definition and properties of characters of homogeneity see [5, articles 20 and 21]. 
we increase $h_{0}$ so that for $h \geqq h_{0}$ the deficiency of the characteristic series of $\left|A_{h}\right|$ will equal $\delta(U)$. Then since $\delta(U)$ is zero, the system $\left|A_{h}\right|$ will cut a complete series on a generic curve of $\left|A_{h}\right|$. It therefore follows that $\left|A_{h r}\right|$ cuts a complete series on a generic curve $A_{h}$ $\in\left|A_{h}\right|$ for $r=1,2, \cdots$, so that $A_{h}$ is arithmetically normal [1].

To show that any nonsingular irreducible hyperplane section of $U_{h}$ is arithmetically normal we observe that the term "generic" is used to signify an irreducible member $A_{h}$ of $\left|A_{h}\right|$ of genus $\pi_{h}$ as was pointed out in footnote 3 . In view of the fact that $\left|A_{h}\right|$ is cut out on $U_{h}$ by the hyperplanes of its ambient space, it is a straightforward matter to show that any two irreducible nonsingular hyperplane sections of $U_{h}$ have the same genus. ${ }^{6}$ It follows that any such member of $\left|A_{h}\right|$ is generic in the sense in which we have used the term, q.e.d.

Models of $\Sigma$ which satisfy the hypothesis of Theorem 2 (that is, normal models $U$ such that $\delta(U)=0$ ) will be called regular models of $\Sigma$. If $\Sigma$ is a regular field, then since $0 \leqq \delta(U) \leqq q$, it follows that every normal model of $\Sigma$ is a regular model. Whether or not irregular fields possess regular models is an open question.

4. Integral bases. Let $\mathrm{D}=k\left[x_{0}, x_{1}, \cdots, x_{n}\right]$ be the integrally closed ring of homogeneous coordinates along an arithmetically normal model $W$ of $\Sigma$. A triple $\left(y_{0}, y_{1}, y_{2}\right)$ of elements of $\mathfrak{D}$ will be called an admissible set of independent variables for $\mathrm{o}$ if (a) $y_{i}$ is homogeneous of degree one, and (b) $D$ is integrally dependent on $k\left[y_{0}, y_{1}, y_{2}\right]$. The elements $y_{0}, y_{1}, y_{2}$ will then be algebraically independent over $k$, and the quotients $y_{1} / y_{0}, y_{2} / y_{0}$ will form a transcendence base for $\Sigma / k$. If $\nu=\left[\Sigma: k\left(y_{1} / y_{0}, y_{2} / y_{0}\right)\right]$, and if there exist $\nu$ elements $\omega_{1}, \omega_{2}, \cdots, \omega_{\nu}$ in $\mathbf{D}$ which are linearly independent over $k\left[y_{0}, y_{1}, y_{2}\right]$ and form a modular base for $\mathrm{o}$ over $k\left[y_{0}, y_{1}, y_{2}\right]$, then the set $\left(y_{0}, y_{1}, y_{2}\right)$ will be called a regular set of independent variables for $\mathrm{o}$. The elements $\omega_{1}, \omega_{2}, \cdots, \omega_{\nu}$ are said to be an independent integral base for $\mathrm{D}$ over $k\left[y_{0}, y_{1}, y_{2}\right]$. The ring $\mathfrak{D}$ will be called a regular ring if every admissible set of independent variables is regular. It is evident that if the set $\left(y_{0}, y_{1}, y_{2}\right)$ is regular then the set $\left(x_{0}, x_{1}, x_{2}\right), x_{i}=\sum a_{i j} y_{j}, a_{i j} \in k,\left|a_{i j}\right| \neq 0$, is also regular.

${ }^{6}$ Let $\mathrm{v}$ be the ring of homogeneous coordinates on $U_{h}$ and let $\chi(n)$ be the number of independent homogeneous elements of degree $n$ in $\mathfrak{D}$. If $A_{h}$ is an irreducible hyperplane section of $U_{h}$ of order $\nu$ and genus $p$, then the fact that the prime ideal of $A_{h}$ in D is a principal ideal, together with the fact that $A_{h}$ is nonsingular, implies that $\chi(n)=2^{-1} n(n-1) \nu+(\nu-p+1) n+c$, where $c$ is a constant. (See [2, article 6] for details.) Since the function $\chi(n)$ is independent of the curve $A_{h}$ used to compute it, the fact that all irreducible nonsingular members of $\left|A_{h}\right|$ have the same genus is established. 
Let $U$ be a regular model of $\Sigma$ and let $U_{h}$ be a derived arithmetically normal model of $U$ such that every irreducible nonsingular hyperplane section of $U_{h}$ is arithmetically normal. Let $\mathfrak{D}$ be the ring of homogeneous coordinates on $U_{h}$ and let $\left(y_{0}, y_{1}, y_{2}\right)$ be an admissible set of independent variables for $\mathfrak{b}$. Since $\mathfrak{o}$ depends integrally on $k\left[y_{0}, y_{1}, y_{2}\right]$, it follows that the ideal $\Sigma_{0} y_{i}$ is irrelevent so that the net of curves, $c_{0} y_{0}+c_{1} y_{1}+c_{2} y_{2}=0, c_{i} \in k$, has no base points on $U_{h}$. Since $U_{h}$ has only a finite number of singularities it follows [9] that the generic curve of this net is nonsingular. Since the quotients $y_{1} / y_{0}$ and $y_{2} / y_{0}$ form a transcendence base for $\Sigma / k$, the general curve of the net is irreducible [7]. Hence, after applying a linear transformation to the quantities $y_{i}$ if necessary, we can assume that (a) the ideals $0 y_{i}$ are prime, (b) the curves $y_{i}=0, i=0,1,2$, intersect pair by pair at simple points of $U_{h}$, and (c) the curves $y_{i}=0$ are arithmetically normal. It is shown in $[2$, article 8$]$ that these conditions are sufficient to insure the existence of an independent integral base for $\mathfrak{D}$ over $k\left[y_{0}, y_{1}, y_{2}\right] .^{7}$ Hence every admissible set of independent variables in $\mathfrak{D}$ is regular, and $\boldsymbol{D}$ is a regular ring. We can therefore assert the following theorem.

THEOREM 3. If $U$ is a regular model of $\Sigma$ then the ring of homogeneous coordinates along a derived arithmetically normal model of $U$ belonging to a sufficiently high character of homogeniety is a regular ring.

5. Regular fields. Let $\Sigma$ be a regular field and let $\xi_{1}, \xi_{2}$ be an arbitrary transcendence base for $\Sigma / k$. The question has been raised by $O$. Zariski as to whether or not the integral closure $\mathfrak{I}$ in $\Sigma$ of the ring $k\left[\xi_{1}, \xi_{2}\right]$ always has an independent integral base over $k\left[\xi_{1}, \xi_{2}\right]$. Although we cannot answer this question, the following theorem throws some light on the problem.

THEOREM 4. If $\left(\xi_{1}, \xi_{2}\right)$ is a transcendence base for the regular field $\Sigma / k$, then there exist integers $h$ such that the integral closure in $\Sigma$ of the ring $R_{h}=k\left[\xi_{1}^{h}, \xi_{2}^{h}\right]$ has an independent integral base over $R_{h}$.

PRoof. For any $h$ the integral closure in $\Sigma$ of $R_{h}$ coincides with the integral closure $\mathfrak{I}$ of $R_{1}$. The ring $\mathfrak{I}$ is a finite integral domain, so that there exist elements $\xi_{3}, \xi_{4}, \cdots, \xi_{n}$ in $\mathfrak{I}$ such that $\mathfrak{I}$ $=k\left[\xi_{3}, \xi_{4}, \cdots, \xi_{n}\right]$. Let $\eta_{0}$ be a transcendental over $\Sigma$ and let $\eta_{i}=\eta_{0} \xi_{i}$, $i=1,2$. If

$$
\text { (5.1) } \xi_{i}^{\nu}+a_{i 1}\left(\xi_{1}, \xi_{2}\right) \xi_{i}^{\nu-1}+\cdots+a_{i \nu}\left(\xi_{1}, \xi_{2}\right)=0, \quad i=3,4, \cdots, n,
$$

" Condition (b) above is somewhat weaker than the corresponding condition (b) given in [2, article 8]. However, the stronger form was used only as a matter of convenience to simplify the details of the proof, as an examination of the proof will show. 
is the equation of integral dependence for $\xi_{i}$ over $R_{1}$ and if $m$ is an integer which is not less than the greatest of the numbers $j^{-1} \cdot \operatorname{deg} a_{i j}$, $i=3,4, \cdots, n ; j=1,2, \cdots, \nu$, then on multiplying (5.1) by $\eta_{0}^{m \nu}$ we find that $\eta_{0}^{m} \cdot \xi_{i}$ depends integrally on $k\left[\eta_{0}, \eta_{1}, \eta_{2}\right]$. By the transitivity of integral dependence it follows that $\eta_{0}^{m} \cdot \xi_{i}$ depends integrally on $k\left[\eta_{0}^{m}, \eta_{1}^{m}, \eta_{2}^{m}\right]$. We let $y_{i}=\eta_{i}^{m}, i=0,1,2 ; y_{j}=\eta_{0}^{m} \cdot \xi_{j}, j=3,4, \cdots, n$. The quantities $y_{0}, y_{1}, \cdots, y_{n}$ can be regarded as the coordinates of the general point of a model $W$ of $\Sigma$. Moreover, the ring $k[y]$ of homogeneous coordinates on $W$ depends integrally on $k\left[y_{0}, y_{1}, y_{2}\right]$.

Let $\rho$ be a character of homogeneity of $W$ and let $U$ be the derived normal model of $W$ belonging to the character $\rho$. Since $\Sigma$ is regular, the model $U$ is regular and hence possesses a derived normal model $U_{\sigma}$ which has a regular ring $\mathfrak{D}=k\left[x_{0}, x_{1}, \cdots, x_{s}\right]$ of homogeneous coordinates. We put $g=\rho \cdot \sigma$ and observe that $g$ is a character of homogeneity of $W$ and that $U_{\sigma}$ is a derived normal model $W_{\sigma}$ of $W$ belonging to the character $g$.

The quantities $y_{0}^{o}, y_{1}^{o}, y_{2}^{o}$ are homogeneous of degree $g$ when the degree is measured with respect to $W$, but they are of degree one when measured with respect to $W_{\boldsymbol{g}}$. These elements are in $\mathfrak{o}$, and it is not difficult to see that every element of $\mathfrak{D}$ depends integrally on $k\left[y_{0}^{o}, y_{1}^{o}, y_{2}^{o}\right]$. In fact, every element of $\boldsymbol{o}$ depends integrally on $k[y]$, so that $\mathfrak{D}$ is integral over $k\left[y_{0}, y_{1}, y_{2}\right]$. Since this latter ring is integral over $k\left[y_{0}^{o}, y_{1}^{o}, y_{2}^{o}\right]$, it follows that $\left(y_{0}^{o}, y_{1}^{o}, y_{2}^{o}\right)$ is an admissible set of independent variables for $\mathfrak{D}$. Since $\mathfrak{D}$ is a regular ring, the set $\left(y_{0}^{g}, y_{1}^{g}, y_{2}^{g}\right)$ is a regular set of independent variables.

After applying a nonsingular linear transformation with coefficients in $k$ we can assume that $x_{i}=y_{i}^{o}, i=0,1,2$. Then $x_{i} / x_{0}=\xi_{i}^{o m}, i=1,2$. If $h=g m$, then the ring $\mathrm{D}_{0}=k\left[x_{1} / x_{0}, x_{2} / x_{0}, \cdots, x_{8} / x_{0}\right]$ depends integrally on $R_{h}$, and since $\mathfrak{D}_{0}$ is integrally closed, $\mathfrak{D}_{0}=\mathfrak{T}$. By $[2$, Theorem 2.1] the fact that $\mathrm{o}$ has an independent modular base over $k\left[x_{0}, x_{1}, x_{2}\right]$ consisting of $\mu=\left[\Sigma: k\left(x_{1} / x_{0}, x_{2} / x_{0}\right)\right]$ elements implies that $\mathrm{D}_{0}$ has an independent integral base over $k\left[x_{1} / x_{0}, x_{2} / x_{0}\right]$, that is, $\mathfrak{I}$ has an independent integral base over $R_{h}$, q.e.d.

\section{BIBLIOGRAPHY}

1. H. T. Muhly, A remark on normal varieties, Ann. of Math. vol. 42 (1941).

2. Integral bases and a characterization of regular surfaces, Trans. Amer. Math. Soc. vol. 54 (1943).

3. H. T. Muhly and O. Zariski, Hilbert's characteristic function and the arithmetic genus of an algebraic variety, to appear in Trans. Amer. Math. Soc.

4. O. Zariski, Algebraic surfaces, Ergebnisse der Mathematik und ihrer Grenzgebiete, vol. 3, 1935.

5. - Some results in the arithmetic theory of algebraic varieties, Amer. J. Math. vol. 61 (1939). 
6. - The reduction of the singularities of an algebraic surface, Ann. of Math. vol. 40 (1939).

7. - Pencils on an algebraic variety and a new proof of a theorem of Bertini, Trans. Amer. Math. Soc. vol. 50 (1941).

8. - A simplified proof for the resolution of singularities of an algebraic surface, Ann. of Math. vol. 43 (1942).

9. - - The theorem of Bertini on the variable singular points of a linear system of varieties, Trans. Amer. Math. Soc. vol. 56 (1944).

UNIVERSITY OF ILLINOIS 